Proceedings of the 6th Polish Symposium of Physics in Economy and Social Sciences (FENS2012), Gdańsk, Poland

\title{
Dynamic Structural and Topological Phase Transitions on the Warsaw Stock Exchange: A Phenomenological Approach
}

\author{
A. Sienkiewicz ${ }^{a}$, T. Gubiec ${ }^{a}$, R. Kutner ${ }^{a, *}$ And Z.R. StruziK ${ }^{b}$ \\ ${ }^{a}$ Institute of Experimental Physics, Faculty of Physics, University of Warsaw Hoża 69, PL-00-681 Warsaw, Poland \\ ${ }^{b}$ The University of Tokyo, Bunkyo-ku, Tokyo 113-0033, Japan
}

\begin{abstract}
We study crash dynamics of the Warsaw Stock Exchange by using minimal spanning tree networks. We identify the transition of the complex network during its evolution from a (hierarchical) power law minimal spanning tree network - representing the stable state of Warsaw Stock Exchange before the recent worldwide financial crash, to a superstar-like (or superhub) minimal spanning tree network of the market decorated by a hierarchy of trees - an unstable, intermediate market state. Subsequently, we observe a transition from this complex tree to the topology of the (hierarchical) power law minimal spanning tree network decorated by several star-like trees or hubs - this structure and topology represent the Warsaw Stock Exchange after the worldwide financial crash, and can be considered to be an aftershock. Our results can serve as an empirical foundation for a future theory of dynamic structural and topological phase transitions on financial markets.
\end{abstract}

DOI: $10.12693 /$ APhysPolA.123.615

PACS: 89.65.Gh, 02.50.Ey, 02.50.Ga, 05.40.Fb, 02.30.Mv

\section{Introduction}

It is only in the past two decades that physicists have intensively studied the structural and/or topological properties of complex networks [1] (and references therein). They have discovered that in most real graphs, small and finite loops are rare and insignificant. Hence, it was possible to assume their architectures to be locally dominated by trees. These properties have been extensively exploited. For instance, it is surprising how well this assumption works in the case of numerous loopy and clustered networks ${ }^{\dagger}$. Therefore, we decided on the minimal spanning tree (MST) technique as a particularly useful, canonical tool in graph theory [2], being a correlation based connected network without any loop [3-10] (and references therein). In the graph, the vertices (nodes) are the companies and the distances between them are obtained from the corresponding correlation coefficients. The required transformation of the correlation coefficients into distances was made according to a simple recipe $[3,5]$.

We consider the dynamics of an empirical complex network of companies, which were listed on the Warsaw Stock Exchange (WSE) for the entire duration of each period of time in question. In general, both the number of companies (vertices) and distances between them can vary in time. That is, in a given period of time these quantities are fixed but in other periods can vary. Obviously, during the evolution of the network some of its edges may disappear, while others may emerge. Hence, neither the number of companies nor edges are conserved quantities. As a result, their characteristics, such as, for

\footnotetext{
*corresponding author; e-mail: Ryszard.Kutner@fuw.edu.pl

${ }^{\dagger}$ Nevertheless, the stability problem of the networks versus their structure and topology should be studied.
}

instance, their mean length and mean occupation layer $[3,6,11-15]$, are continuously varying over time, as discussed below.

We applied the MST technique to find the transition of a complex network during its evolution from a hierarchical (power law) tree representing the stock market structure before the recent worldwide financial crash [16] to a superstar-like tree (superhub) decorated by a hierarchy of trees (hubs), representing the market structure during the period of the crash. Subsequently, we found the transition from this complex tree to the power law tree decorated by the hierarchy of local star-like trees or hubs (where the richest of these hubs could be a candidate for another superhub) representing the market structure and topology after the worldwide financial crash.

We foresee that our results, being complementary to others obtained earlier [17-20], can serve as a phenomenological foundation for the modeling of dynamic structural and topological phase transitions and critical phenomena on financial markets [21].

\section{Results and discussion}

The initial state (graph or complex network) of the WSE is shown in Fig. 1 in the form of a hierarchical $\mathrm{MST}^{\ddagger}$. This graph was calculated for $N=142$ companies present on WSE for the period from 2005-01-03 to 2006-03-09, i.e. before the worldwide financial crash occurred [16].

\footnotetext{
$\ddagger$ For the construction of the MST network, we here used Prim’s algorithm [22], which is quicker than Kruskal's algorithm [22, 23], in particular for the number of companies $N \gg 1$. Both algorithms are often used.
} 


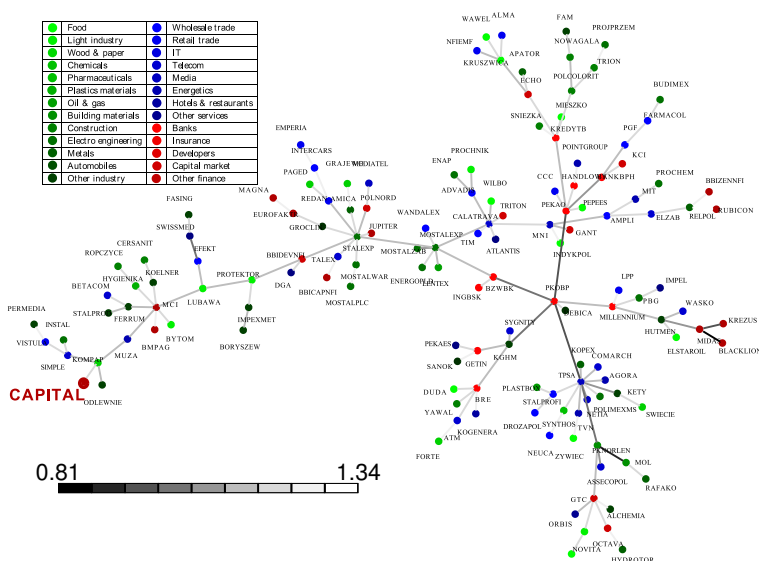

Fig. 1. The hierarchical MST associated with the WSE (and consisting of $N=142$ companies) for the period from 2005-01-03 to 2006-03-09, before the worldwide financial crash. The companies are indicated by the colored circles (see the legend for an additional description). We focus on the financial company CAPITAL Partners (large red circle), as later it plays a central role in the MST, shown in Fig. 2. When the link between two companies is in dark grey, the cross-correlation between them is greater, while the distance between them is shorter (cf. the corresponding scale incorporated there). However, the geometric distances between companies, shown in the figure by the lengths of straight lines, are arbitrary, otherwise the tree would be much less readable.

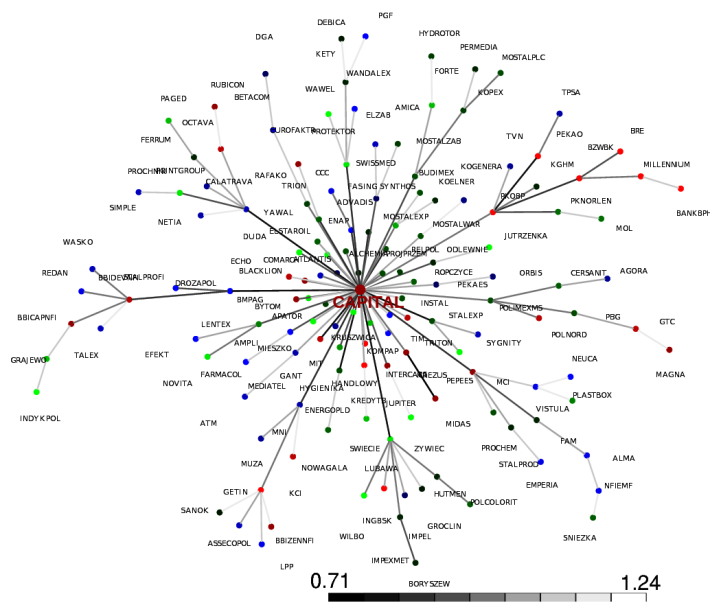

Fig. 2. The superstar-like graph (or superhub) of the MST (also consisting of $N=142$ companies of the WSE) observed for the period from 2007-06-01 to 2008$-08-12$, which covers the worldwide financial crash. Now CAPITAL Partners becomes a dominant hub (or superhub). It is a temporal giant component of the MST, i.e. the central company of the WSE.
We focus on the financial company CAPITAL Partners ${ }^{\S}$. It is a marginal company for the most of the period in question. However, it becomes a central company for the MST presented in Fig. 2, for the period from 2007-06-01 to $2008-08-12$, which covers the worldwide financial crash.

In other words, for this period of time, CAPITAL Partners is represented by a vertex which has a much larger number of edges (or it is of a much larger degree) than any other vertex (or company). This means that it becomes a dominant hub (superhub) or a giant component of the MST.

In the way described above, the transition between two structurally and topologically different states of the stock exchange is realized. We observe the transition from hierarchical (power law) MST (consisting of a hierarchy of local stars or hubs) to the superstar-like (or superhub) MST decorated by the hierarchy of trees (hubs).

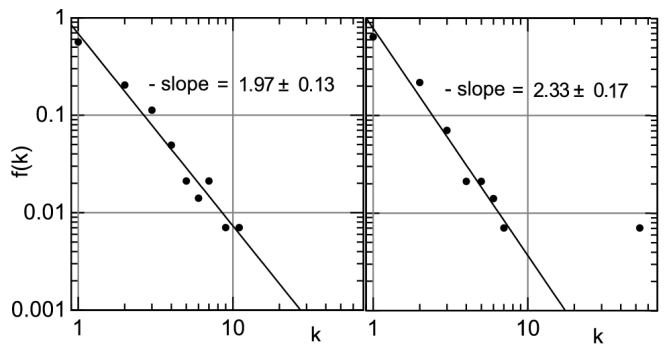

Fig. 3. The comparison of power law discrete distributions $f(k)$ vs. $k$ (where $k$ is the vertex degree) for the hierarchical MST shown in Fig. 1 and the superstar-like MST decorated by the hierarchy of trees shown in Fig. 2. One can observe that for the latter MST there is a single vertex (rhs plot), which has a degree much larger (equaling 53) than any other vertex. This vertex represents the company CAPITAL Partners, which is a superextreme event or a dragon king [25-28], being a giant component of the MST network [1].

In Fig. 3 we compare discrete distributions of vertex degrees ${ }^{\top}$. Although the distributions obtained are power laws, we cannot say that we are here dealing with a Barabási-Albert (BA) type of complex network with their rule of preferential linking of new vertices [24]. This is because for both our trees, the power law exponents are distinctly smaller than 3 (indeed, the exponent equal to 3 characterizes the BA network), which is a typical observation for many real complex networks [1].

Remarkably, the right hand side (rhs) plot in Fig. 3 makes it possible to consider the tree presented in Fig. 2

$\S$ CAPITAL Partners was listed on the WSE from 20 October 2004. The main activities of the company are capital investment in various assets and investment advice.

T The discrete distribution of the vertex degree is normalized by a factor equal to the total number of vertices $N$ fixed for a given period of time. 
as a power law MST decorated by a temporal dragon king $\|$. This is because the single vertex (representing CAPITAL Partners) is located far from the straight line (in the $\log -\log$ plot) and can be considered as a temporally outstanding, superextreme event or a temporal dragon king [25-28], which condenses most of the edges (or links). Hence, the probability $f\left(k_{\max }\right)=0.007=$ $1 / 142$, where $k_{\max }=53$ is the degree of the dragon king (which is the maximum degree here). We suggest that the appearance of such a dragon king could signify a precursor of a crash**.

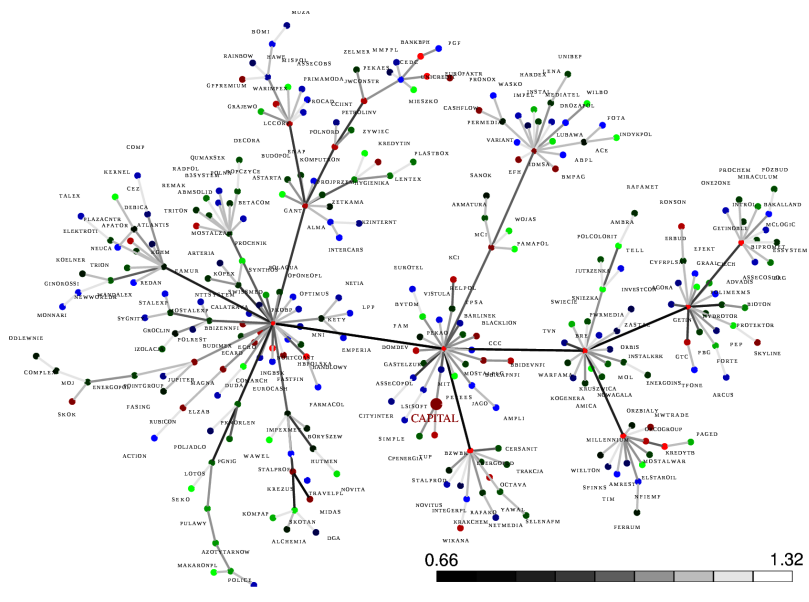

Fig. 4. The hierarchical graph of the MST decorated by several local star-like trees for the WSE for the period from 2008-07-01 to 2011-02-28, that is after the worldwide financial crisis. The companies are indicated by colored circles (see the legend in Fig. 1). The CAPITAL company is no longer the central hub, but has again become a marginal company (vertex). When the link between two companies is in dark grey, the cross-correlation between them is greater, while the distance between them is shorter. However, the geometric distances between companies, shown in the figure by the length of the straight lines, are arbitrary, otherwise the tree would be much less readable.

For completeness, the MST was constructed for $N=$ 274 companies of the WSE for a third period of time from 2008-07-01 to 2011-02-28, i.e. after the worldwide financial crash (cf. Fig. 4). It is interesting that several new (even quite rich) hubs appeared while the single superhub (superstar) disappeared (as it became a marginal vertex). This means that the structure and topology of the network strongly vary during its evolution over the market crash. This is also well confirmed by the plot

"The equivalent terms "superextreme event" and "dragon king" stress that [25]: (i) we are dealing with an exceptional event which is completely different in comparison with the usual events; (ii) this event is significant, being distinctly outside the power law. For instance, in paper [26] sustained and impetuous dragon kings were defined and discussed.

${ }^{* *}$ This promising hypothesis requires a more systematic study. in Fig. 5, where several points (representing large hubs) are located above the power law. This power law is defined by the slope equal to $-2.62 \mp 0.18$ and cannot be considered as a BA complex network. Rather, it is analogous to the structure and topology of the Internet, which is characterised by almost the same slope [29, 30]. It would be an interesting project to identify the actual local dynamics (perhaps nonlinear) of our network, which subsequently creates and then annihilates the temporal singularity (i.e. the temporal dragon king).

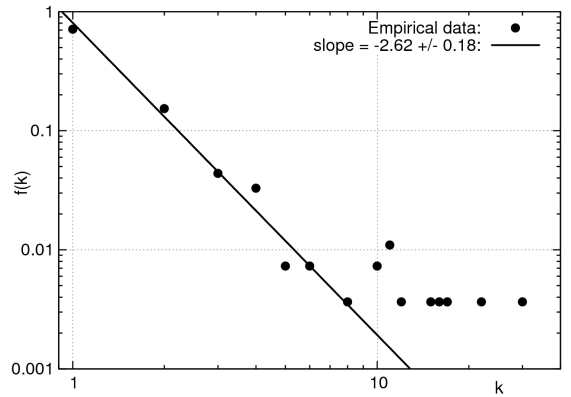

Fig. 5. The power law discrete distribution $f(k)$ vs. $k$ (where $k$ is the vertex degree) for the MST shown in Fig. 4. Six points (associated with several different companies) appear above the power law. This means that several large hubs appear instead of a single superhub. The richest vertex here has the degree $k_{\max }=30$ and the corresponding probability $f\left(k_{\max }\right)=0.0036=1 / 274$. However, this vertex cannot be considered to be a superextreme event (or dragon king) because it is not separated far enough from other vertices.

The considerations given above are confirmed in the plots shown in Figs. 6 and 7. The well-defined absolute minima at the beginning of 2008 of the normalized length (in Fig. 6) and mean occupation layer (in Fig. 7) vs. time are clearly visible.

As usual [13-15], the normalized length of the MST network simply means the average length of the edge directly connecting two vertices ${ }^{\dagger \dagger}$. This normalized length vs. time has an absolute minimum close to 1 at the beginning of 2008 (cf. Fig. 6), while at other times much shallower (local) minima are observed. This result indicates the existence of a more compact structure at the beginning of 2008 than at other times.

Furthermore, by applying the mean occupation layer defined, as usual [13-15], by the mean number of subsequent edges connecting a given vertex of a tree with the central vertex (here CAPITAL Partners), we obtained similar results (cf. the solid curve in Fig. 7). For comparison, the result based on the other central temporal hubs (having currently the largest degrees) was also obtained (cf. the dotted curve in Fig. 7). All the approaches used

\footnotetext{
$\dagger^{\dagger}$ Obviously, the edge between two vertices is taken into account only if a connection between them exists.
} 


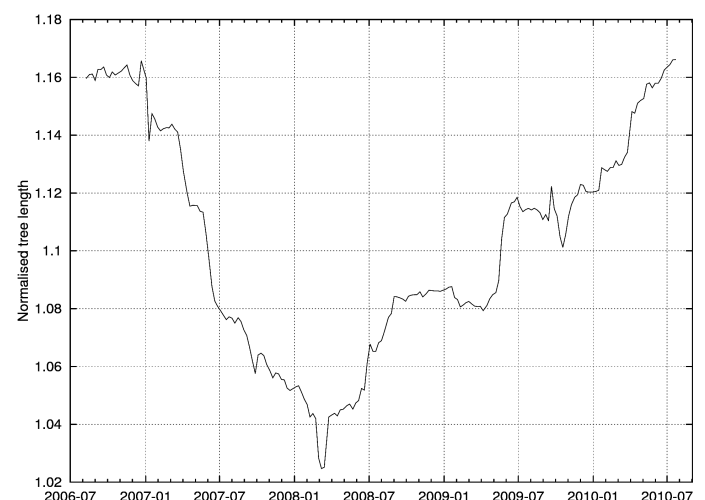

Fig. 6. Normalized length of the MST vs. time (counted in trading days (td)). The well-defined absolute minimum of the curve is located at the beginning of 2008. This localization (in the period from 2007-06-01 to $2008-08-12$, covering the crash), together with the corresponding length so close to 1.0 , confirm the existence of a network, which is significantly more compact than others.

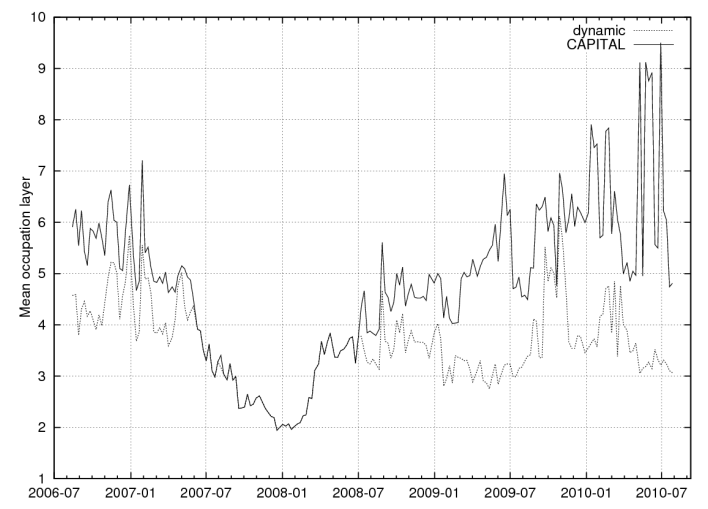

Fig. 7. Mean occupation layer for the MST vs. time (counted in trading days, (td)), where CAPITAL Partners was assumed to be the central hub (the solid curve). For comparison, the result based on the central temporal hubs (currently having the largest degrees, the dotted curve) was obtained. The well-defined absolute minimum, common for both curves, is located at the beginning of 2008 (in the period from 2007-06-01 to 2008$-08-12)$.

above give fully consistent results. In particular, both curves in Fig. 7 coincide in the period from 2007-06-01 to 2008-08-12, having a common absolute minimum located at the beginning of 2008. To plot the dotted curve, the company, associated with the largest degree, is chosen at each time as a temporal central hub. In general, such a company is, from time to time, replaced by another company. However, for the period indicated above the CAPITAL Partners has largest degree (while other companies of course, have smaller degrees). This observation is confirmed by the behavior of the solid curve constructed by assuming CAPITAL Partners to be a fixed company central hub. Just outside this period, CAPI-
TAL Partners is no longer a central hub (again becoming a peripheral company) as other companies assume the role of the central hub. The well defined region of overlap between the two curves in Fig. 7, defines our second period of the transition dynamics (from 2007-06-01 to 2008-08-12). This results from the observation that the dotted curve in Fig. 7 is placed below the solid one outside this interval period. For this reason, the dynamically unstable period identified ends on 12 August, 2008. This does not include the period between September 2008 and March 2009, where the most serious drawdown during the worldwide financial crash 2007-2009 occurred. Some precursor of the crash may be contained here in the unstable state of the WSE - this hypothesis is to be investigated in our subsequent work.

The existence of the absolute minimum (shown in Fig. 7) for CAPITAL Partners, and simultaneously the existence of the absolute minimum shown in Fig. 6 in the first quarter of 2008 (to a satisfactory approximation) confirms the existence of the star-like structure (or superhub), as a giant component of the MST, centered around CAPITAL Partners. We may suppose that the evolution from a marginal to the central company of the stock exchange and again to a marginal company is stimulated by the most attractive financial products offered by this company to the market only in the second period of time (i.e. in the period from 2007-06-01 to 2008-08-12).

\section{Concluding remarks}

In this work, we have studied the empirical evolving connected correlated network associated with a small size stock exchange, the WSE. Our results identify the surprising fact that a marginal capitalization company like CAPITAL Partners (less than one permil of a typical WIG20 company) becomes a dominant hub (superhub) in the dynamically unstable period (see Fig. 2 for details).

Our work provides empirical evidence that there is a dynamic structural and topological first order phase transition in the time range dominated by a crash. Namely, before and after this range the superhub disappears and we observe the power law MST and power law MST decorated by several hubs ${ }^{\ddagger \ddagger}$, respectively before and after the unstable state of the WSE. Therefore, our results consistently confirm the existence of the dynamic structural and topological phase transitions, which can be roughly summarized as follows:

phase of power law MST - a stable state

$\Rightarrow$ phase of the superstar-like structure or superhub MST decorated by hierarchy of trees or hubs — a likely unstable state

$\ddagger \ddagger$ The best candidate for the superhub within the third period (from 2008-07-01 to 2011-02-28) could be, perhaps, PKOBP (see Figs. 4 and 5 for details), which is a richest vertex having degree equal to 30 . 
$\Rightarrow$ phase of power law MST decorated by several star-like trees or hubs (where the richest hub could be a candidate for another superhub) a likely stable state.

We assume the hypothesis that the first transition can be considered as a signature of a stock exchange crash, while the second one can be understood to be an aftershock. Nevertheless, the second transition related to the third period requires a more detailed analysis. Indeed, in this period PKOBP resembles a superhub (see Figs. 4 and 5 for details), which could reflect other stable state of the WSE. This possibly indicates that we are dealing with an indirect transition (of the first order type) between two stable components, with a distinct unstable component in between.

One of the most significant observations contained in this work is shown in the plots in Figs. 3 and 5. Namely, the exponents of all the degree distributions are smaller than 3, which means that all variances of vertex degrees diverge. This indicates that we are here dealing with criticality as the range of fluctuations is comparable with the size of the graph. This further indicates that the network evolution from 2005-01-03 to 2011-02-28 takes place within the scaling region ${ }^{\S \S}[1,31-33]$ containing a critical point. Apparently, we are here dealing with scale-free networks, which are ultrasmall worlds 9 ฯ [34-36].

Similar results have been obtained by us for a larger size stock market, the Frankfurt Stock Exchange [in preparation]. In fact, we obtained results analogous to those presented in all our Figs. 1-7, where the role of the dynamically unstable central hub (or superhub) is played by the GITTARSALZ AG Stahl und Technologie company.

Our results are also complementary to those obtained earlier by Drożdż et al. [19]. Their results focused on the slow (stable) component (state). Namely, they constructed the MST network of 1000 highly capitalized American companies. The topology of this MST shows its centralization around the most important stable node, General Electric. This was found both using binary and weighted MSTs.

In this context, it should be stressed that the discontinuous (i.e. the first order) phase transitions evolve towards the continuous (i.e. second order) phase transitions. The discontinuous phase transition transcends over possible unstable (superhub) states, reminiscent of unstable supercooled or superheated states of matter. Such possible unstable states cannot be considered to be noise*** in the

\footnotetext{
$\S \S$ Note that the scaling region is a region where signatures of both the first order and of the second order phase transitions are present together. Here we suppose that this is, indeed, the case.

ฯ For the ultrasmall world, the mean length between two vertices of a graph is proportional to $\ln \ln N$ instead of that for the small world proportional only to $\ln N$.

*** Indeed, the case of the noise has been discussed in this context in paper [37].
}

system. Rather they should be considered as an integral part of the dynamics and reflect the natural evolution of the system until the critical point is reached (cf. [20] and references therein, where the role of stable states (or slow components) on NYSE or NASDAQ was considered by using binary and weighted MSTs).

We consider that the phenomenological theory of cooperative phenomena in networks proposed by Goltsev et al. [38] (based on the concepts of the Landau theory of continuous phase transitions) could be a promising approach. However, the analytical treatment of the dynamics of such a network based phase transition phenomena remains a challenge. An alternative view of our results could consider the superhub phase as a temporal condensate [1] (and references therein). Hence, we can reformulate the phase transitions mentioned above to represent the dynamic transition from the disordered phase to a temporal condensate, and then the transition from the condensate again to some disordered phase.

We consider our work to be a good starting point for finding similar topological transitions in other markets. We can summarize this work with the conclusion that it could be promising to study in detail the phase transitions considered above, which can define an empirical basis for understanding of stock market evolution as a whole.

\section{Acknowledgments}

We are grateful to Rosario N. Mantegna and Tiziana Di Matteo for their helpful comments and suggestions. This work was partially supported by the Grant No. 119 awarded within the First Competition of Economic Research organized by the National Bank of Poland.

\section{References}

[1] S.N. Dorogovtsev, A.V. Goltsev, J.F.F. Mendes, Rev. Mod. Phys. 80, 1275 (2008).

[2] B. Bollobás, Modern Graph Theory, Springer, Berlin 1998.

[3] R.N. Mantegna, Eur. Phys. J. B 11, 193 (1999).

[4] G. Bonanno, G. Calderelli, F. Lillo, S. Micciche, N. Vandewalle, R.N. Mantegna, Eur. Phys. J. B 38, 363 (2004).

[5] R.N. Mantegna, H.E. Stanley, An Introduction to Econophysics. Correlations and Complexity in $\mathrm{Fi}$ nance, Cambridge University Press, Cambridge 2000.

[6] G. Bonanno, F. Lillo, R.N. Mantegna, Quant. Fin. 1, 96 (2001).

[7] N. Vandewalle, F. Brisbois, X. Tordoir, Quant. Fin. 1, 372 (2001).

[8] L. Kullmann, J. Kertész, K. Kaski, Phys. Rev. E 66, 026125 (2002).

[9] M. Tumminello, T. Di. Matteo, T. Aste, R.N. Mantegna, Eur. Phys. J. B 55, 209 (2007).

[10] M. Tumminello, C. Coronello, F. Lillo, S. Micciche, R.N. Mantegna, Int. J. Bifurc. Chaos 17, 2319 (2007). 
[11] J.G. Brida, W.A. Risso, Expert Syst. Appl. 37, 3846 (2010).

[12] B.M. Tabak, T.R. Serra, D.O. Cajueiro, Eur. Phys. J. $B$ 74, 243 (2010)

[13] J.-P. Onnela, A. Chakraborti, K. Kaski, J. Kertész, Eur. Phys. J. B 30, 285 (2002).

[14] J.-P. Onnela, A. Chakraborti, K. Kaski, J. Kertész, Physica A 324, 247 (2003).

[15] J.-P. Onnela, A. Chakraborti, K. Kaski, J. Kertész, A. Kanto, Phys. Rev. E 68, 056110 (2003).

[16] D. Sornette, Why Stock Markets Crash, Princeton University Press, Princeton 2003.

[17] A. Fronczak, P. Fronczak, J.A. Holyst, Eur. Phys. J. B 59, 133 (2007).

[18] A. Fronczak, P. Fronczak, J.A. Holyst, Phys. Rev. E 70, 056110 (2004)

[19] S. Drożdż, J. Kwapień, J. Speth, AIP Conf. Proc 1261, 256 (2010).

[20] J. Kwapień, S. Drożdż, Phys. Rep. 515, 115 (2012).

[21] W. Weidlich, G. Haag, Concepts and Models of a Quantitative Sociology. The Dynamics of Interacting Populations, Springer-Verlag, Berlin 1983.

[22] D.B. West, Introduction to Graph Theory, Prentice-Hall, Englewood Cliffs 1996.

[23] J.B. Kruskal, Proc. Am. Math. Soc. 7, 48 (1956).

[24] R. Albert, A.-L. Barabási, Rev. Mod. Phys. 74, 47 (2001).

[25] D. Sornette, Int. J. Terraspace Eng. 2, 1 (2009).

[26] T. Werner, T. Gubiec, R. Kutner, D. Sornette, Eur. Phys. J. Special Topics 205, 27 (2012).

[27] Extreme Events in Nature and Society, Eds. S. Albeverio, V. Jentsch, H. Kantz, Springer-Verlag, Berlin 2006.

[28] Y. Malevergne, D. Sornette, Extreme Financial Risks. From Dependence to Risk Management, Springer-Verlag, Berlin 2006.
[29] M. Faloutsos, P. Faloutsos, Ch. Faloutsos, in: $S I G-$ COMM'99, Proc. Conf. on Applications, Technologies, Architectures, and Protocols for Computer Communications, Vol. 29, Eds. L. Chapin, J.P.G. Sterbenz, G. Parulkar, J. Turner, P. Skelly, E. Zegura, Harvard University, Science Center, Cambridge (MA) 1999, p. 251.

[30] Q. Chen, H. Chang, R. Govindan, S. Jamin, S.J. Shenker, W. Willinger, in: Proc. 21st Annual Joint Conf. of the IEEE Computer and Communications Societies, IEEE Computer Society, New York 2002, p. 608.

[31] D. Sornette, Critical Phenomena in Natural Sciences. Chaos, Fractals, Selforganization and Disorder: Concepts and Tools, 2nd ed., Springer Series in Synergetics, Springer-Verlag, Heidelberg 2004.

[32] R. Badii, A. Politi, Complexity. Hierarchical Structures and Scaling in Physics, Cambridge University Press, Cambridge 1997.

[33] P. Hohenberg, B. Halperin, Rev. Mod. Phys. 59, 435 (1977).

[34] D.J. Watts, S.H. Strogatz, Collective-Dynamics of "Small-World" Networks, Princeton University Press, Princeton 1999.

[35] L.A.N. Amaral, A. Scala, M. Barthelemy, H.E. Stanley, Proc. Natl. Acad. Sci. USA 97, 11149 (2000).

[36] R. Cohen, S. Havlin, Phys. Rev. Lett. 90, 058701 (2003).

[37] D.-M. Song, M. Tumminello, W.-X. Zhou, R.N. Mantegna, Phys. Rev. E 84, 026108 (2011).

[38] A.V. Goltsev, S.N. Dorogovtsev, J.F.F. Mendes, Phys. Rev. E 67, 026123 (2003). 